\title{
Domain analysis reveals striking functional differences between the regulatory subunits of phosphatidylinositol 3-kinase (PI3K), p85a and $p 85 \beta$
}

\author{
Yoshihiro Ito ${ }^{1}$, Peter K. Vogt ${ }^{1}$ and Jonathan R. Hart ${ }^{1}$ \\ ${ }^{1}$ Department of Molecular Medicine, The Scripps Research Institute, La Jolla, San Diego, CA, USA \\ Correspondence to: Peter K. Vogt, email: pkvogt@scripps.edu \\ Keywords: SH2 domain, SH3 domain, RhoGAP domain, iSH2 domain, domain exchange \\ Received: November 11,2016 Accepted: July 12,2017 Published: August 03, 2017 \\ Copyright: Ito et al. This is an open-access article distributed under the terms of the Creative Commons Attribution License 3.0 (CC BY 3.0), \\ which permits unrestricted use, distribution, and reproduction in any medium, provided the original author and source are credited.
}

\section{ABSTRACT}

Our understanding of isoform-specific activities of phosphatidylinositol 3-kinase (PI3K) is still rudimentary, and yet, deep knowledge of these non-redundant functions in the PI3K family is essential for effective and safe control of PI3K in disease. The two major isoforms of the regulatory subunits of PI3K are p85a and p85 $\beta$, encoded by the genes PIK3R1 and PIK3R2, respectively. These isoforms show distinct functional differences that affect and control cellular PI3K activity and signaling [1-4]. In this study, we have further explored the differences between $\mathrm{p} 85 \mathrm{a}$ and $\mathrm{p} 85 \beta$ by genetic truncations and substitutions. We have discovered unexpected activities of the mutant proteins that reflect regulatory functions of distinct p85 domains. These results can be summarized as follows: Deletion of the SH3 domain increases oncogenic and PI3K signaling activity. Deletion of the combined SH3-RhoGAP domains abolishes these activities. In p85 $\beta$, deletion of the $\mathrm{CSH} 2$ domain reduces oncogenic and signaling activities. In p85a, such a deletion has an activating effect. The deletions of the combined $\mathrm{CSH} 2$ and iSH2 domains and also the deletion of the $\mathrm{CSH} 2$, iSH2 and nSH2 domains yield results that go in the same direction, generally activating in p85a and reducing activity in $\mathrm{p} 85 \beta$. The contrasting functions of the $\mathrm{CSH} 2$ domains are verified by domain exchanges with the $\mathrm{CSH} 2$ domain of $\mathrm{p} 85 \beta$ exerting an activating effect and the cSH2 domain of p85a an inactivating effect, even in the heterologous isoform. In the cell systems studied, protein stability was not correlated with oncogenic and signaling activity. These observations significantly expand our knowledge of the isoform-specific activities of $p 85 a$ and $p 85 \beta$ and of the functional significance of specific domains for regulating the catalytic subunits of class IA PI3K.

\section{INTRODUCTION}

We recently reported an oncogenic activity of wild type $\mathrm{p} 85 \beta$ in cultures of avian embryonic fibroblasts [2]. This activity manifests itself in cellular transformation that alters cell morphology and increases the growth potential of the cells. It is also correlated with enhanced PI3K signaling as documented by elevated phosphorylation of AKT at S473. Wild type p85 $\alpha$ lacks such oncogenic activity. The causes for this distinct difference in the biological and biochemical properties of these two p85 isoforms are not known. The $\mathrm{p} 85 \alpha$ and $\mathrm{p} 85 \beta$ proteins are encoded by two different genes, PIK3R1 and PIK3R2, but show extensive protein sequence homology. Both consist of five structurally and functionally defined domains. They are, starting at the N-terminus: an $\mathrm{SH} 3$ domain, a RhoGAP domain (also referred to as breakpoint-cluster region homology $(\mathrm{BH})$ domain that is flanked by two proline-rich regions), an N-terminal $\mathrm{SH} 2$ domain (nSH2), an inter-SH2 region (iSH2), and a C-terminal SH2 (cSH2) domain $[1,3]$. The $\mathrm{p} 85$ proteins can interact with a multitude of proteins involved in cellular signaling $[1,3]$. The most important interactions are an inhibitory binding of the $\mathrm{nSH} 2$ domain to the helical domain of the catalytic subunit p110 of PI3K and, for class IA PI3Ks, activating interactions of the $\mathrm{SH} 2$ domains with upstream signaling 
proteins containing targeted phosphotyrosine residues [59].

Here we have investigated the biological and biochemical activities of $\mathrm{p} 85$ domains comparing the $\alpha$ and $\beta$ isoforms. The results provide new insights into the functions of the p85 domains. These include an inhibitory action of the $\mathrm{SH} 3$ domain and essential roles of the RhoGAP and cSH2 domains in oncogenic signaling. These studies also reveal functional differences between the $\mathrm{p} 85 \alpha$ and $\mathrm{p} 85 \beta \mathrm{cSH} 2$ domains; the hinge domain located between iSH2 and cSH2 contributes to these differences.

\section{RESULTS}

\section{The SH3 domain of p85 inhibits PI3K signaling}

We cloned two N-terminal truncations of both the human PIK3R1 and PIK3R2 genes; in one, the SH3 domain was deleted, in the other, both $\mathrm{SH} 3$ and RhoGAP domains were deleted (Figure 1A). These constructs were expressed with the RCAS vector in chicken embryo fibroblasts
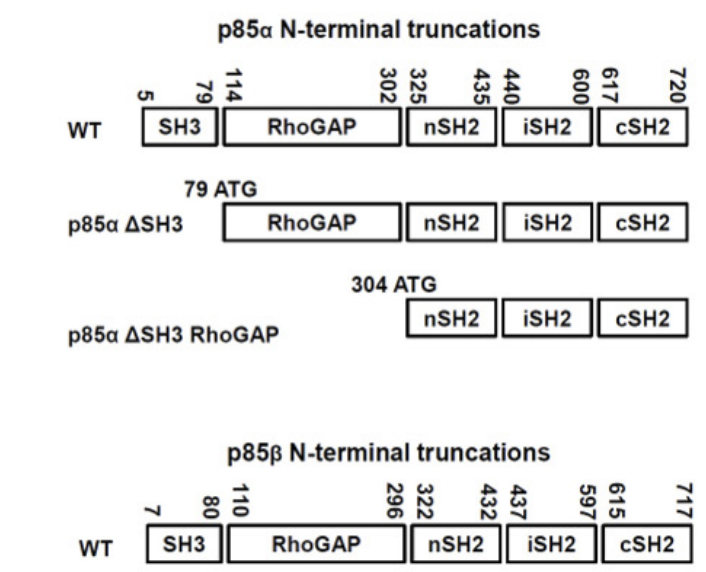

\begin{tabular}{|c|c|c|c|c|}
\hline \multicolumn{5}{|c|}{105 ATG } \\
\hline $\mathrm{p} 85 \beta \Delta \mathrm{SH} 3$ & RhoGAP & $\mathrm{nSH} 2$ & iSH2 & $\mathrm{cSH} 2$ \\
\hline \multicolumn{5}{|c|}{314 ATG } \\
\hline $85 \beta \Delta \mathrm{SH}_{3}$ & & $\mathrm{nSH} 2$ & iSH2 & $\mathrm{cSH} 2$ \\
\hline
\end{tabular}

A

SHOGAP
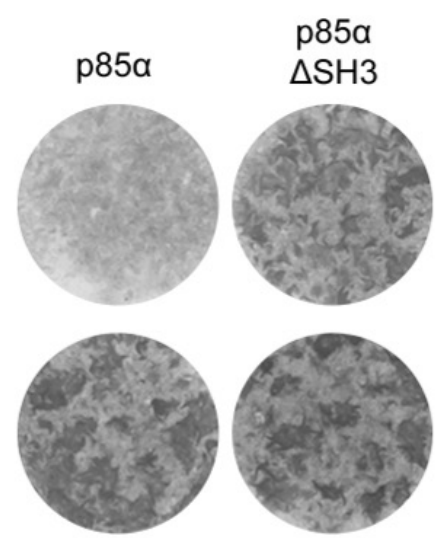

B

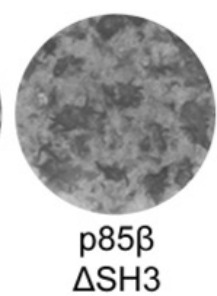

p85a $\triangle \mathrm{SH} 3$

RhoGAP
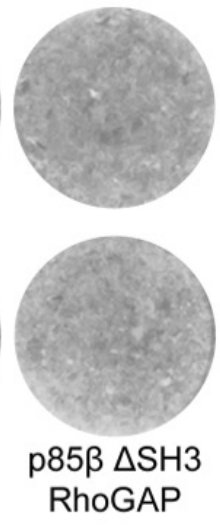
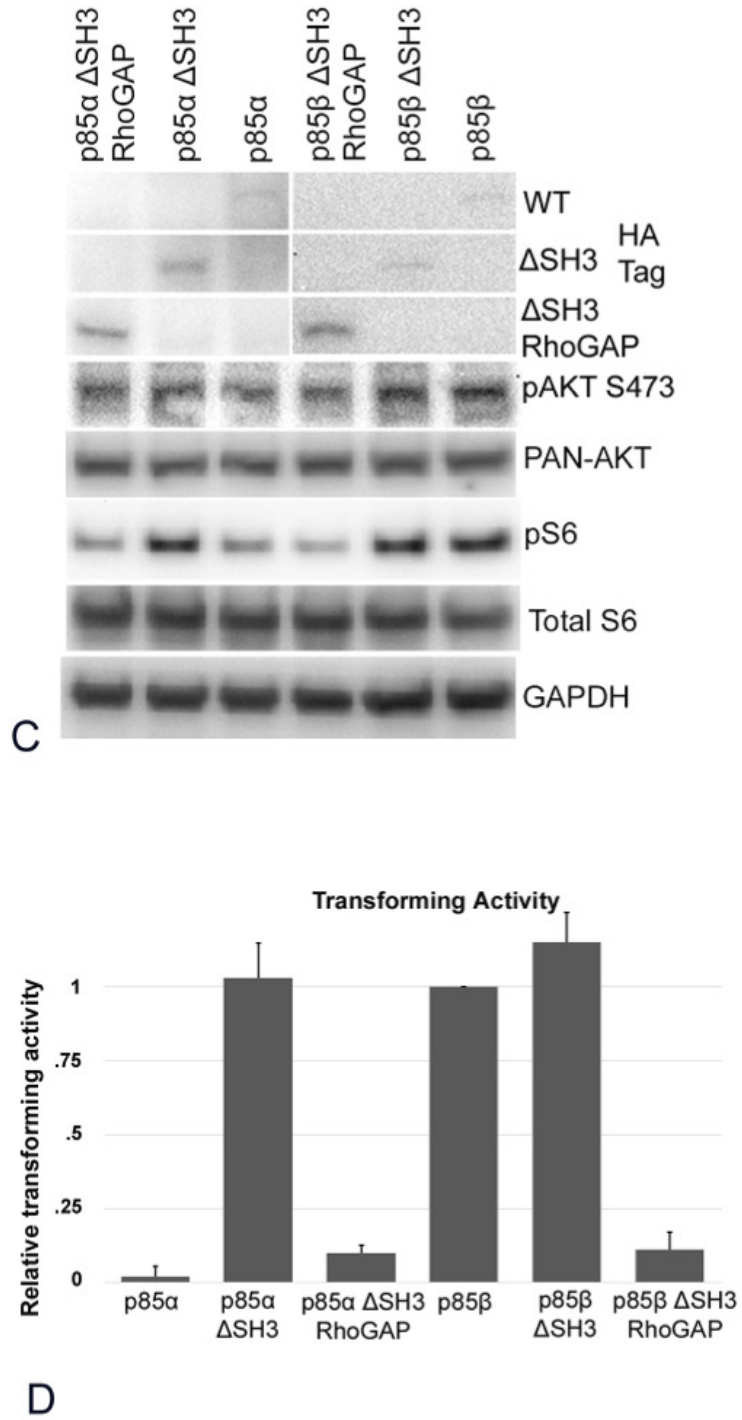

Figure 1: N-terminal truncations of p85. A. Schematic representation of the p $85 \mathrm{~N}$-terminal truncation mutants. B. Representative focus assays of $\mathrm{p} 85 \mathrm{~N}$-terminal truncations. C. Activation of PI3K signaling in cells expressing N-terminally truncated p 85 as reflected by Western blots of Akt phosphorylated at S437 and S6 at Ser235/236. The data in Figure 1C are from a representative experiment that was repeated three times with consistent results (for details, see the Materials and Methods section). D. Efficiencies of cellular transforming activity of $\mathrm{p} 85 \mathrm{~N}$-terminal truncation mutants relative to the transforming activity of wild type $\mathrm{p} 85 \beta$. CEF cells were transfected RCAS(A) constructs expressing the inserts $\mathrm{p} 85 \beta, \mathrm{p} 85 \beta \Delta \mathrm{SH} 3, \mathrm{p} 85 \beta \Delta \mathrm{SH} 3$-RhoGAP, $\mathrm{p} 85 \alpha, \mathrm{p} 85 \alpha \Delta \mathrm{SH} 3$, and $\mathrm{p} 85 \alpha \Delta \mathrm{SH} 3$-RhoGAP. Transforming activity was standardized to $0.5 \mu \mathrm{g}$ transfected DNA. 
(CEF). Expression of the SH3 domain truncation of both $\mathrm{p} 85 \alpha(\mathrm{p} 85 \alpha \Delta \mathrm{SH} 3)$ and $\mathrm{p} 85 \beta(\mathrm{p} 85 \beta \Delta \mathrm{SH} 3)$ increased the formation of oncogenically transformed cell foci as compared with the unmodified constructs (Figure 1B and 1D). However, the truncation of both $\mathrm{SH} 3$ and RhoGAP domains in both p85 isoforms (p85 $\triangle$ SH3-RhoGAP) resulted in a lack of oncogenic activity (Figure $1 \mathrm{~B}$ and 1D). We also determined the level of PI3K signaling in p85 $\triangle \mathrm{SH} 3$, wild type $\mathrm{p} 85$ and the p85 $\triangle \mathrm{SH} 3$-RhoGAP expressing CEF for both isoforms. Signaling was determined by Western blotting using the phosphorylation of AKT at S473 and of S6 at Ser235/236 as markers. Compared to cells transfected with wild type $\mathrm{p} 85$, the SH3 truncations showed increased phosphorylation of AKT and of S6. No such increase was detected with the $\triangle \mathrm{SH} 3-\mathrm{RhoGAP}$ deletions (Figure 1C). These data show that a deletion of the $\mathrm{SH} 3$ domain enhances both signaling and oncogenic activity of the p85 isoforms. The SH3 domain contains a proline-rich motif that in $\mathrm{p} 85 \alpha$ has been reported to be a necessary mediator for efficient binding to PTEN. The interaction between PTEN and $p 85 \alpha$ protects PTEN from proteolytic degradation $[10,11]$ and the p85a C-terminal truncations

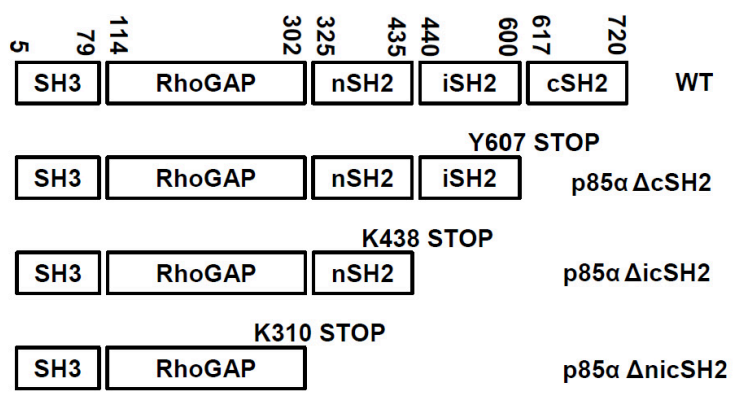

A

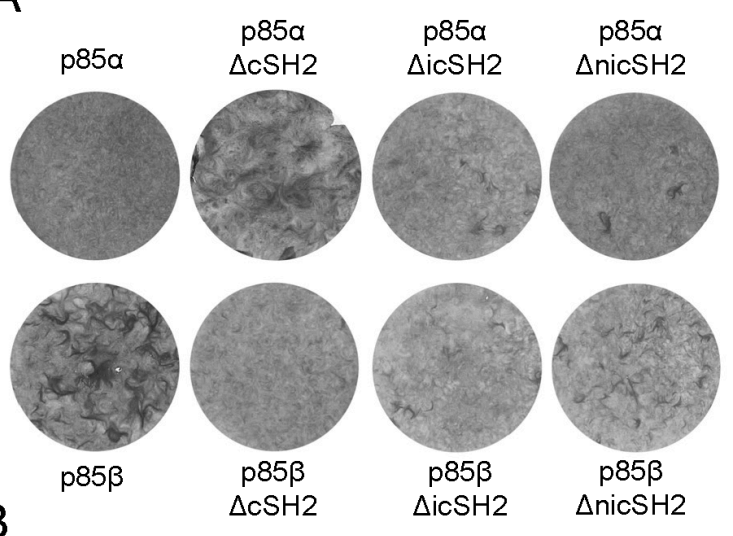

B

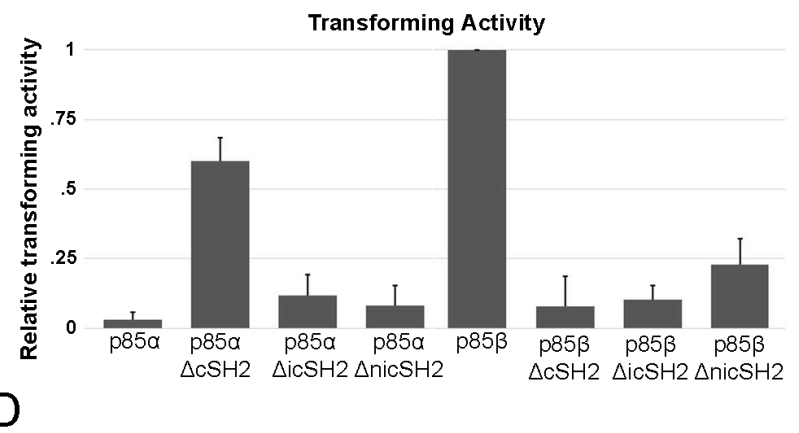

p85 $\beta$ C-terminal truncations

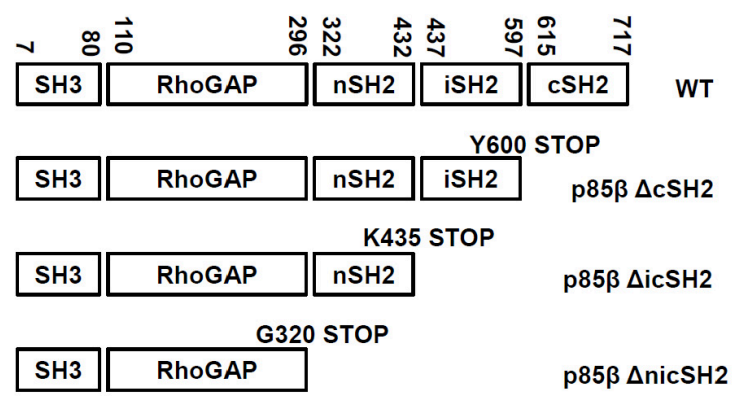

Figure 2: C-terminal truncations of p85. A. Schematic representation of the $\mathrm{p} 85 \mathrm{C}$-terminal truncation mutants. B. Representative focus assays of $\mathrm{p} 85 \mathrm{C}$-terminal truncations. C. PI3K signaling in cells expressing C-terminally truncated p85 as reflected by Western blots of Akt phosphorylated at S437 and S6 phosphorylated at Ser235/236. The data in Figure 2C are from a representative experiment that was repeated three times with consistent results (for details, see the Materials and Methods section). D. Efficiencies of cellular transforming activity of $\mathrm{p} 85 \mathrm{C}$-terminal truncation mutants relative to the transforming activity of wild type $\mathrm{p} 85 \beta$. CEF were transfected with RCAS(A) constructs expressing the inserts $\mathrm{p} 85 \beta, \mathrm{p} 85 \beta \Delta \mathrm{cSH} 2, \mathrm{p} 85 \beta \Delta \mathrm{icSH} 2, \mathrm{p} 85 \beta \Delta \mathrm{nicSH} 2, \mathrm{p} 85 \alpha, \mathrm{p} 85 \alpha \Delta \mathrm{cSH} 2, \mathrm{p} 85 \alpha \Delta \mathrm{icSH} 2$, and $\mathrm{p} 85 \alpha \Delta \mathrm{nicSH} 2$. Transforming activity was standardized to $0.5 \mu \mathrm{g}$ transfected DNA. 
SH3 deletion would prevent that protection and result in increased PI3K activity. Our data suggest that the SH3 domain in both p85 isoforms exerts an inhibitory effect on PI3K signaling. In the case of $\mathrm{p} 85 \alpha$, this could reflect the known interaction with PTEN; in the case of $\mathrm{p} 85 \beta$, the mechanism of SH3-mediated inhibition remains to be identified.

\section{The cSH2 domains of $p 85 \alpha$ and of $p 85 \beta$ show opposite effects in the regulation of PI3K oncogenic signaling}

We also analyzed C-terminal truncations of the $\mathrm{p} 85$ isoforms for their transforming and signaling activities. These constructs are depicted in Figure 2A and include

p85 domain swapping

\begin{tabular}{|c|c|c|c|c|c|}
\hline p85a & $\mathrm{SH} 3$ & RhoGAP & nSH2 & iSH2 & cSH2 \\
\hline$p 85 \beta$ & $\mathrm{SH} 3$ & RhoGAP & nSH2 & iSH2 & cSH2 \\
\hline $\mathrm{p} 85 \alpha / \beta \mathrm{iSH} 2$ & $\mathrm{SH} 3$ & RhoGAP & nSH2 & iSH2 & cSH2 \\
\hline 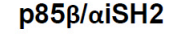 & $\mathrm{SH} 3$ & RhoGAP & nSH2 & iSH2 & cSH2 \\
\hline $\mathrm{p} 85 \alpha / \beta \mathrm{cSH} 2$ & SH3 & RhoGAP & nSH2 & iSH2 & cSH2 \\
\hline p85ß/acSH2 & SH3 & RhoGAP & nSH2 & iSH2 & cSH2 \\
\hline
\end{tabular}

A

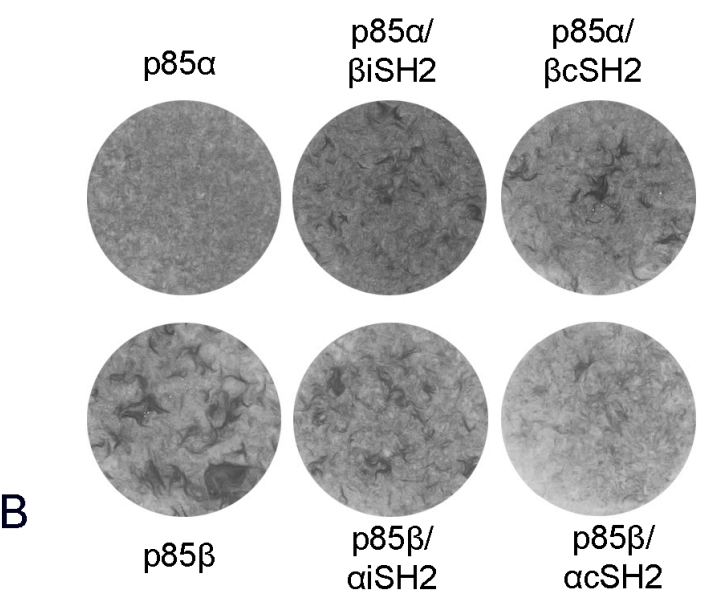

A deletions of the cSH2 domain (p85 $\Delta \mathrm{cSH} 2$ ), the joint truncation of the iSH2 and $\mathrm{cSH} 2$ domains (p85 $\Delta \mathrm{icSH} 2)$ and deletion of the $\mathrm{nSH} 2$, iSH2 and $\mathrm{cSH} 2$ domains (p85 $\Delta$ nicSH2). It is known that a deletion of the cSH2 domain can enhance signaling activity of $\mathrm{nSH} 2$ mutants of $\mathrm{p} 85 \alpha$, and spontaneously occurring C-terminal truncations of $p 85 \alpha$ activate the oncogenic potential of $p 85 \alpha[2,12]$ and of $p 85 \beta$ [13]. Here we show that for $\mathrm{p} 85 \alpha$, truncation of the $\mathrm{cSH} 2$ domain by itself can activate focus-forming activity compared to the inactive wild type version of the protein (Figure 2B and 2D). This activation is correlated with enhanced phosphorylation of AKT S473 (Figure $2 \mathrm{C})$. In contrast, the homologous truncation in $\mathrm{p} 85 \beta$ reduces focus-forming activity compared to wild type and attenuates AKT phosphorylation in CEF (Figure 2B - 2D). These observations suggest that the $\mathrm{cSH} 2$ domain of $\mathrm{p} 85 \alpha$

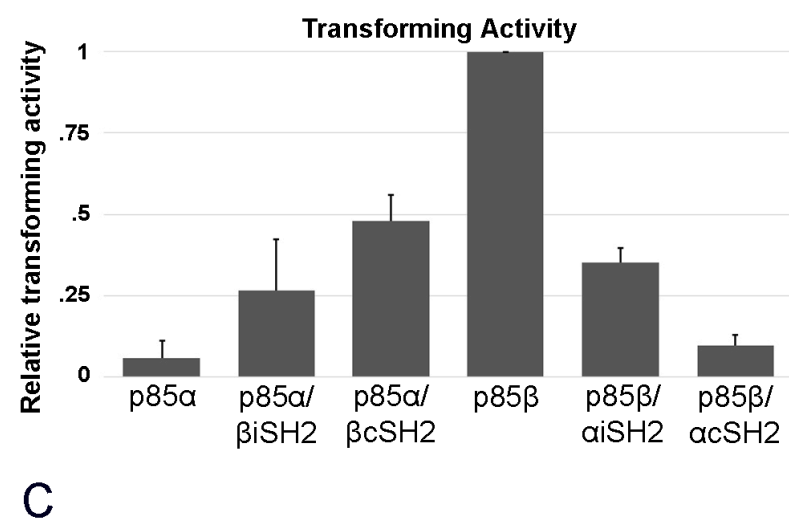

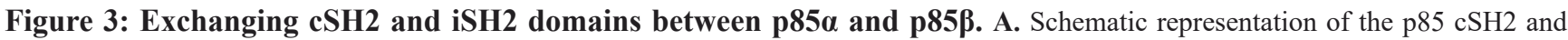
iSH2 domain exchange mutants. B. Representative focus assays of p85 C-terminal domain exchange mutants. C. Efficiencies of cellular transforming activity of $\mathrm{p} 85$ domain exchange mutants relative to the transforming activity of wild type p85 $\beta$. CEF were transfected with $\mathrm{RCAS}(\mathrm{A})$ constructs expressing the inserts $\mathrm{p} 85 \beta, \mathrm{p} 85 \alpha, \mathrm{p} 85 \beta / \alpha \mathrm{cSH} 2, \mathrm{p} 85 \beta / \alpha \mathrm{iSH} 2, \mathrm{p} 85 \alpha / \beta \mathrm{cSH} 2$, and $\mathrm{p} 85 \alpha / \beta \mathrm{SSH} 2$. Transforming activity was standardized to $0.5 \mu \mathrm{g}$ transfected DNA. D. PI3K signaling in cells expressing p 85 domain exchange mutants as reflected by Western blots of S6 phosphorylated at Ser235/236. The data in Figure 3D are from a representative experiment that was repeated three times with consistent results (for details, see the Materials and Methods section). 
acts as a negative regulating element for PI3K signaling, whereas in $\mathrm{p} 85 \beta$, the $\mathrm{cSH} 2$ makes a positive contribution to the oncogenic and signaling activities of the wild

$\begin{array}{ll}\text { p85a hinge: } & \text { ENTEDQYSLVEDDEDLP } \\ \text { p85 } \beta \text { hinge: } & \text { KNETEDQYALMEDEDDLP }\end{array}$

A

p85 domain swapping

\begin{tabular}{|c|c|c|c|c|c|}
\hline \multirow[b]{2}{*}{ p85a } & \multirow[b]{2}{*}{$\mathrm{SH} 3$} & \multirow[b]{2}{*}{ RhoGAP } & \multirow[b]{2}{*}{ nSH2 } & \multicolumn{2}{|c|}{ Hinge } \\
\hline & & & & iSH2 & cSH2 \\
\hline p & SH3 & RhoGAP & nSH2 & iSH2 & cSH2 \\
\hline
\end{tabular}

\begin{tabular}{|c|c|c|c|c|c|}
\hline p85a/h $\beta \mathrm{cSH} 2$ & $\mathrm{SH} 3$ & RhoGAP & $\mathrm{nSH} 2$ & iSH2 & cSH2 \\
\hline$p 85 \beta / h \alpha$ & $\mathrm{SH} 3$ & RhoGAP & nSH2 & iSH2 & $\mathrm{CSH} 2$ \\
\hline
\end{tabular}

B
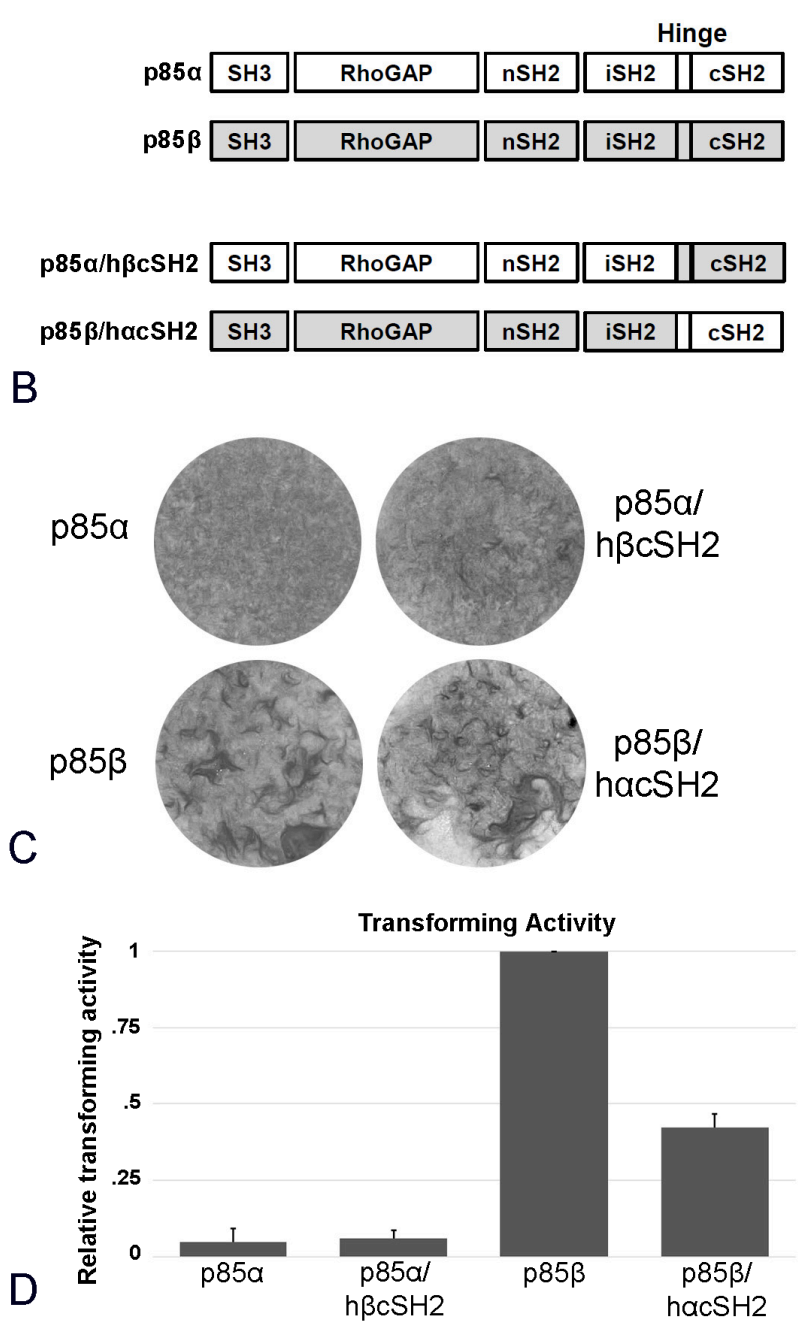

type protein. Deleting both the iSH2 and cSH2 domains $(\triangle \mathrm{icSH} 2)$ reduced the activating effect of the $\mathrm{cSH} 2$ deletion in p $85 \alpha$ and yielded a marginally active construct

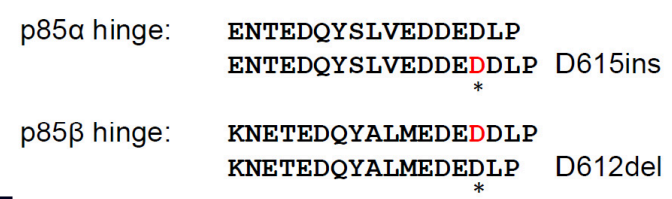

$\mathrm{E}$
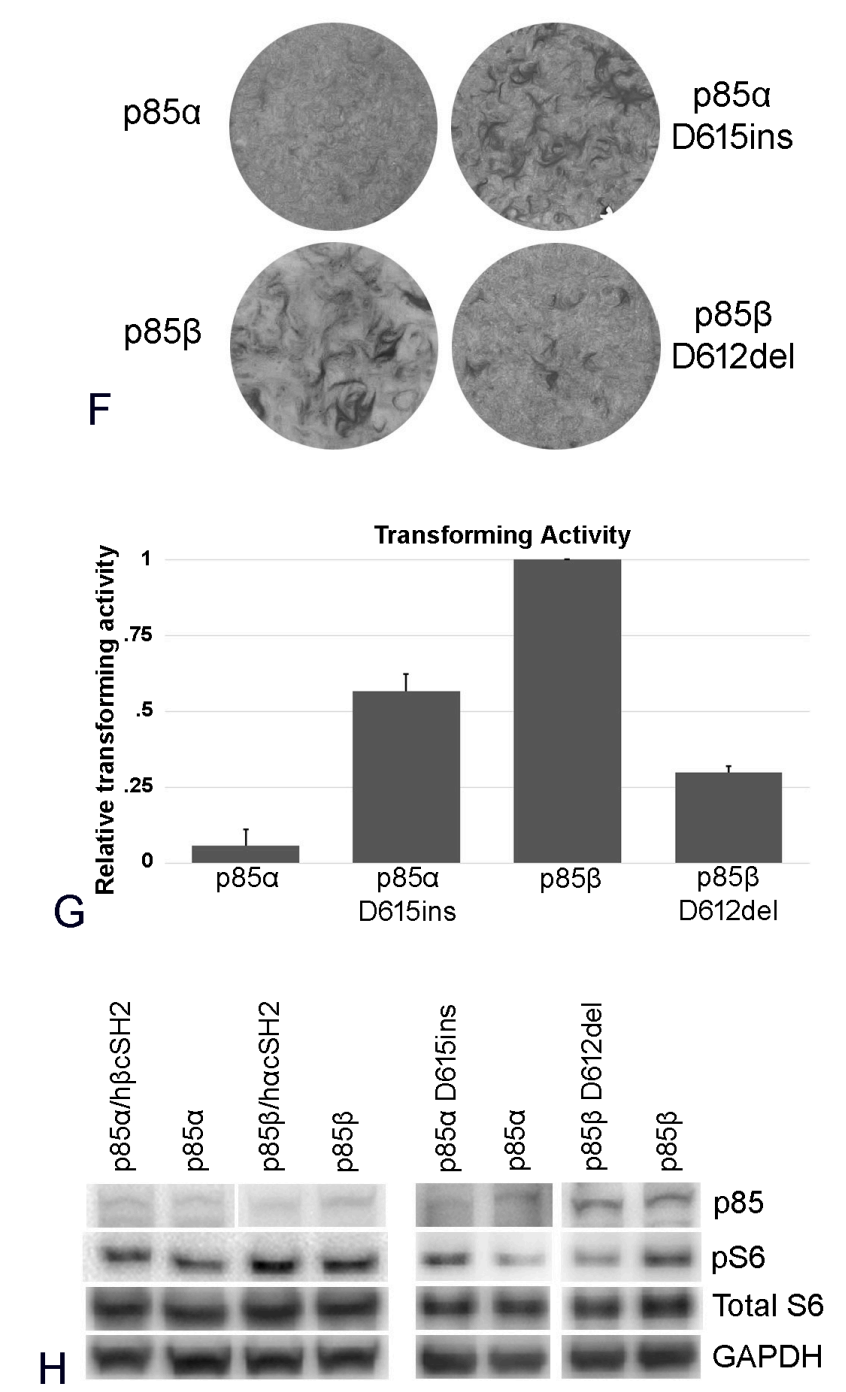

Figure 4: The effect of changing the p85 hinge region located between iSH2 and cSH2. A. Comparison of the amino acid sequences of the hinge regions of $\mathrm{p} 85 \alpha$ and $\mathrm{p} 85 \beta$. B. Schematic representation of the $\mathrm{p} 85$ hinge region constructs. C. Representative focus assays of $\mathrm{p} 85 \alpha$ and $\mathrm{p} 85 \beta$ wild type and of the hinge region-cSH2 exchange constructs depicted in B. D. Efficiencies of cellular transforming activity of p85 hinge region constructs relative to the transforming activity of wild type p85 $\beta$. CEF were transfected with RCAS(A) constructs expressing $\mathrm{p} 85 \beta, \mathrm{p} 85 \alpha, \mathrm{p} 85 \beta / \mathrm{h} \alpha \mathrm{cSH} 2, \mathrm{p} 85 \alpha / \mathrm{h} \beta \mathrm{cSH} 2$ ). Transforming activity was standardized to $0.5 \mu \mathrm{g}$ transfected DNA. E. Sequence of single amino acid insertions and deletion in the p85 hinge regions (p85 $\alpha$ D615ins and p85 $\beta$ D612del). F. Representative focus assays of single amino acid insertions or deletions in the hinge region of p85. G. Efficiencies of cellular transforming activity of p85 single amino acid insertion or deletion mutant constructs relative to the transforming activity of wild type p85 $\beta$. CEF were transfected with RCAS(A) constructs expressing p $85 \alpha, \mathrm{p} 85 \beta$, p85 $\alpha$ D615ins, and p85 3 D612del. Transforming activity was standardized to $0.5 \mu \mathrm{g}$ transfected DNA. H. PI3K signaling in cells expressing p 85 hinge region constructs and the single-amino acid insertion or deletion mutants as reflected by Western blots of S6 phosphorylated at Ser235/236. The data in Figure $4 \mathrm{H}$ are from a representative experiment that was repeated three times with consistent results (for details, see the Materials and Methods section). 
in the case of $\mathrm{p} 85 \beta$ (Figure $2 \mathrm{~B}$ and $2 \mathrm{D}$ ). The constructs expressing the triple truncation of the $\mathrm{nSH} 2$, iSH2 and cSH2 domains $(\Delta$ nicSH2) were not significantly different in activity from the aforementioned double truncations.
Exchange of cSH2 and of iSH2 domains between p85 $\alpha$ and $p 85 \beta$ further documents functional differences between the two isoforms

We then exchanged the $\mathrm{cSH} 2$ and the iSH2 domains of $\mathrm{p} 85 \alpha$ and $\mathrm{p} 85 \beta$ (Figure 3A). The $\mathrm{cSH} 2$ and iSH2 domains of $p 85 \beta$ induce oncogenic activity in $p 85 \alpha$; in
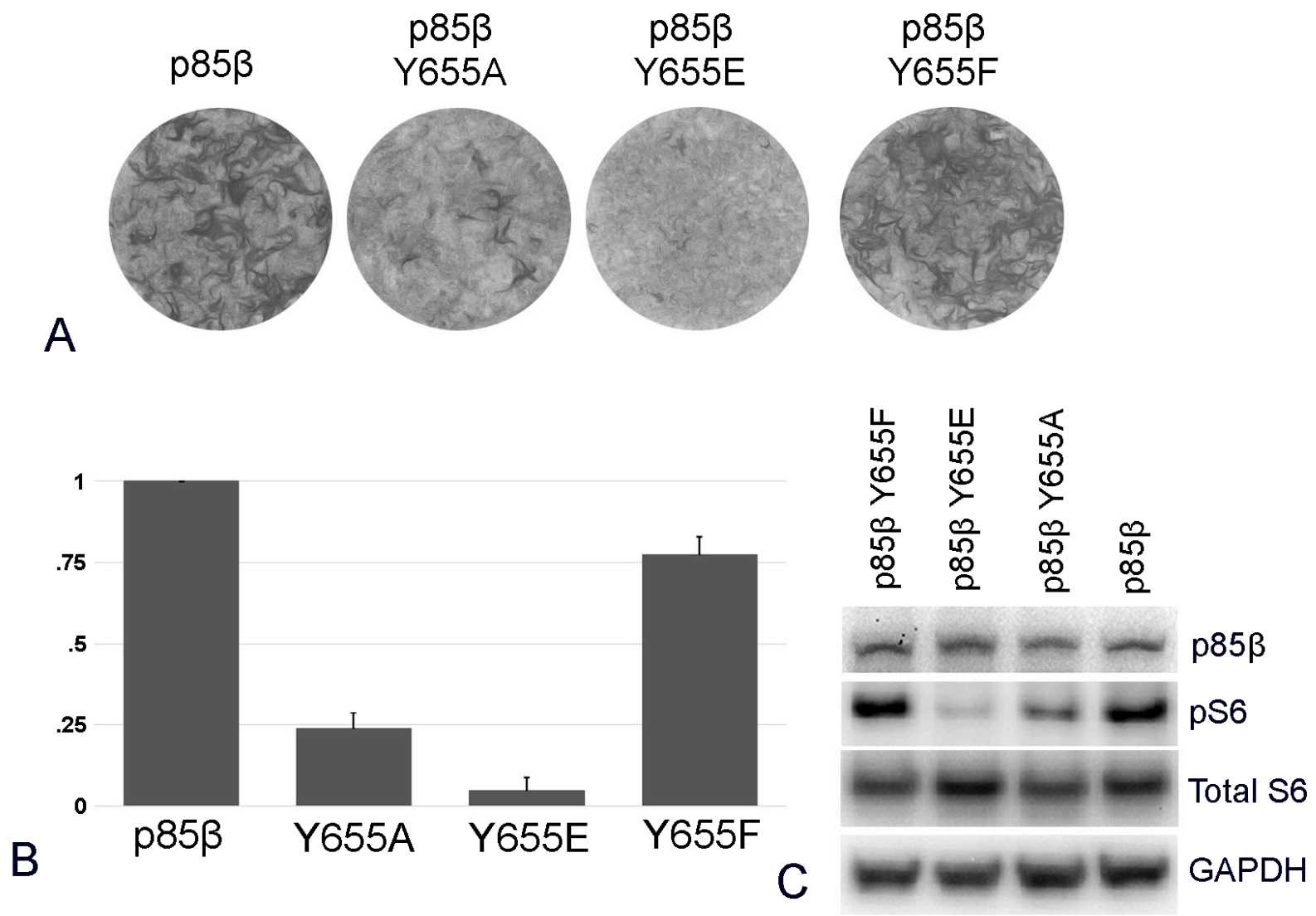

\section{Cycloheximide $(50 \mu \mathrm{g} / \mathrm{ml})$ treatment}
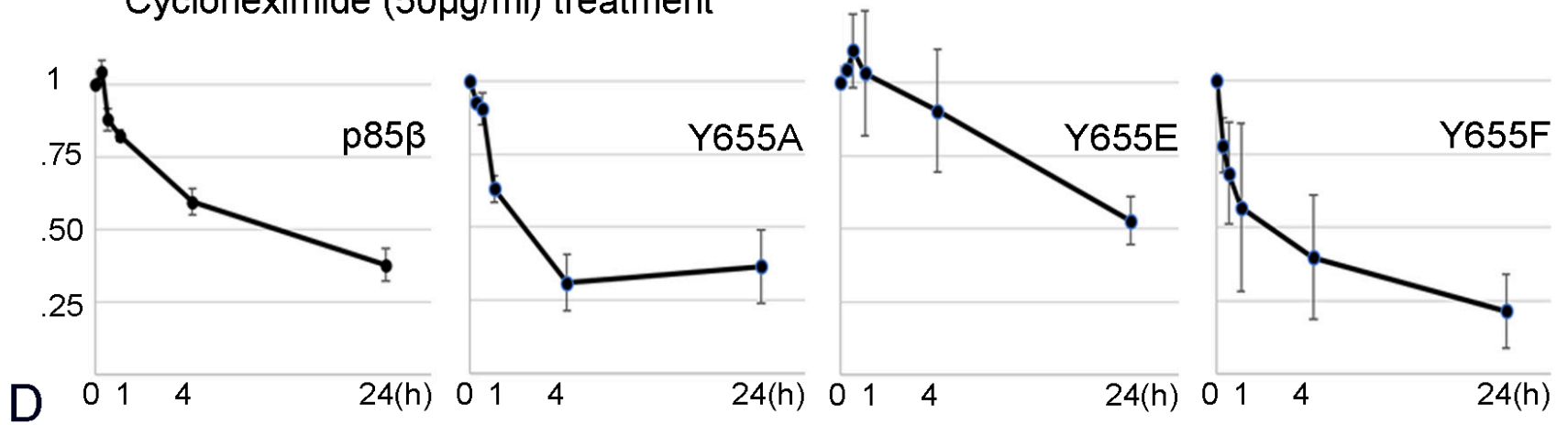

Figure 5: p85 $\beta$ protein stability and oncogenic activity. A. Representative focus assays of p85 $\beta$ and p $85 \beta$ Y 655 mutants. B. Efficiencies of cellular transforming activity of p85 Y655 mutants relative to the transforming activity of p85 $\beta$. CEF were transfected RCAS(A) construct expressing $\mathrm{p} 85 \beta$ and the point mutations. Transforming activity was standardized to $0.5 \mu \mathrm{g}$ transfected DNA. C. PI3K signaling in cells expressing p $85 \beta$ and p $85 \beta$ Y 655 mutants as reflected by Western blots of S6 phosphorylated at Ser235/236. The data in Figure 5C are from a representative experiment that was repeated three times with consistent results (for details, see the Materials and Methods section). D. Time course of degradation of $\mathrm{p} 85 \beta$ and its Y655 mutant proteins as determined by cycloheximide $(50 \mu \mathrm{g} / \mathrm{ml})$ treatment. Protein concentrations were determined by Western blotting. 
contrast, wild type p $85 \alpha$ has only very low focus-forming ability, and even this may not reflect wild type proper but spontaneously occurring activating C-terminal deletions [12] (Figure 3B and 3D). These domain exchanges did not appear to enhance signaling by $\mathrm{p} 85 \alpha$ as reflected in AKT phosphorylation. However, the base value generated by wild type $\mathrm{p} 85 \alpha$ probably reflects the occurrence of spontaneous activating mutations that routinely occur during retroviral expression of that protein. These make it impossible to rule out small yet significant differences between wild type and exchange mutant signaling [12] (Figure $3 \mathrm{C}$ ). In contrast to these positive regulatory activities of the $\mathrm{p} 85 \beta$ domains, the $\mathrm{p} 85 \alpha \mathrm{cSH} 2$ and iSH2 domains have a negative effect on the oncogenic actions of $\mathrm{p} 85 \beta$ (Figure 3B - 3D). The cSH2 domain of $\mathrm{p} 85 \alpha$ almost completely abolished the focus-forming activity of wild type $\mathrm{p} 85 \beta$ (Figure 3B and 3D). These observations expand the functional differences between $\mathrm{p} 85 \alpha$ and $\mathrm{p} 85 \beta$ to the iSH2 domain. These differences probably reflect distinct interactions with $\mathrm{p} 110$ or with regulatory proteins.

\section{The different hinge regions between the iSH2 and cSH2 domains of $p 85 \alpha$ and $p 85 \beta$ attenuate the activities of the $\mathrm{cSH} 2$ regions}

The iSH2 and cSH2 domains of $\mathrm{p} 85 \alpha$ and $\mathrm{p} 85 \beta$ are linked by a hinge region (Figure $4 \mathrm{~A}$ ). In $\mathrm{p} 85 \alpha$, the
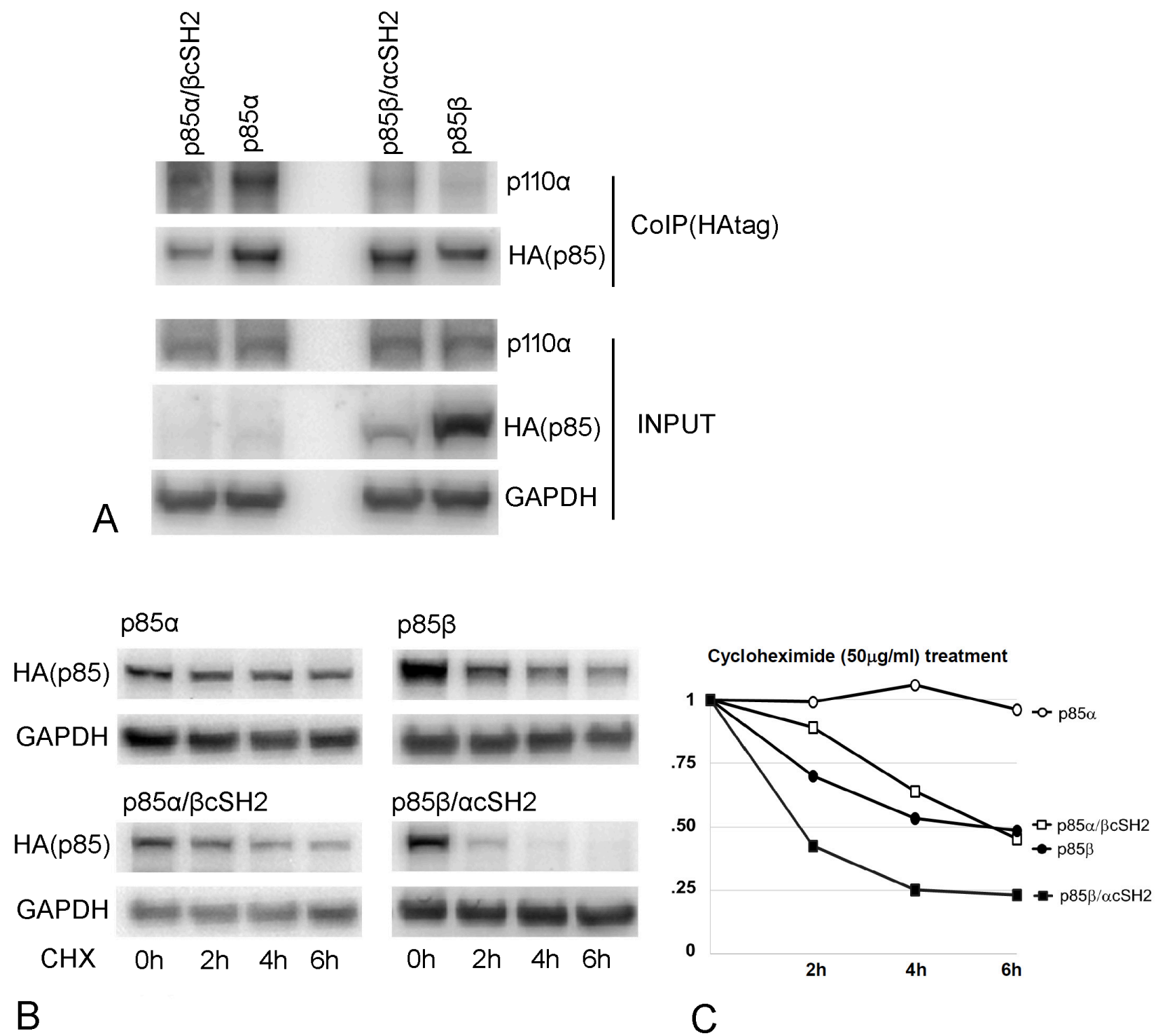

Figure 6: Protein interactions and stability of p85 cSH2 exchange mutants. A. Co-immunoprecipitation of p110 $\alpha$ with HA-tagged $\mathrm{p} 85 \beta, \mathrm{p} 85 \beta / \alpha \mathrm{cSH} 2, \mathrm{p} 85 \alpha$ and $\mathrm{p} 85 \alpha / \beta \mathrm{cSH} 2$. p110 $\alpha$ interacts with wild type $\mathrm{p} 85 \alpha, \mathrm{p} 85 \beta$ and the cSH2 exchange mutants. B. Cycloheximide $(50 \mu \mathrm{g} / \mathrm{ml})$ treatment of $\mathrm{p} 85 \alpha, \mathrm{p} 85 \beta$ and the $\mathrm{cSH} 2$ exchange mutants. The Western blot indicates the protein expression level during cycloheximide $(50 \mu \mathrm{g} / \mathrm{ml})$ treatment compared to GAPDH as a control. C. Time course of p85 and exchange mutant degradation as determined by cycloheximide treatment. 
hinge region contains the residue that is the target of phosphorylation by p110 (S608). Phosphorylation of S608 results in downregulation of PI3K activity [14]. The hinge region of $p 85 \beta$ lacks this phosphorylation site and therefore does not mediate this auto-inhibition. The hinge regions of $\mathrm{p} 85 \alpha$ and $\mathrm{p} 85 \beta$ also differ in length: In $\mathrm{p} 85 \alpha$, the region extends to 17 amino acids and in $\mathrm{p} 85 \beta$ to 18 amino acids (Figure 4A). In order to test for possible effects of these differences in sequence and length, we constructed $\mathrm{cSH} 2$ exchange mutants with the respective hinge region included $(\mathrm{p} 85 \alpha / \mathrm{h} \beta \mathrm{cSH} 2$ and $\mathrm{p} 85 \beta / \mathrm{h} \alpha \mathrm{cSH} 2$, Figure 4B). We also carried out one-amino acid deletions and substitutions in the respective hinge regions ( $\mathrm{p} 85 \alpha$ D615ins and p85 $\beta$ D612del, Figure 4F). Exchanging cSH2 domains plus hinge region had a significantly smaller effect on oncogenic activity compared to the exchange of the cSH2 domains alone (Figure 4C and 4D vs. Figure 3A, $3 \mathrm{~B}$ and $3 \mathrm{D}$ ). The length of the hinge region also appeared to have a mild effect in that adding an amino acid to the p85 $\alpha$ hinge enhances oncogenic activity, and deleting an amino acid from the hinge region of $\mathrm{p} 85 \beta$ decreases focus formation (Figure 4F). We conclude that the strong functional differences of the $\mathrm{p} 85 \alpha$ and $\mathrm{p} 85 \beta \mathrm{cSH} 2$ domains can be significantly reduced by the hinge region between iSH2 and cSH2.
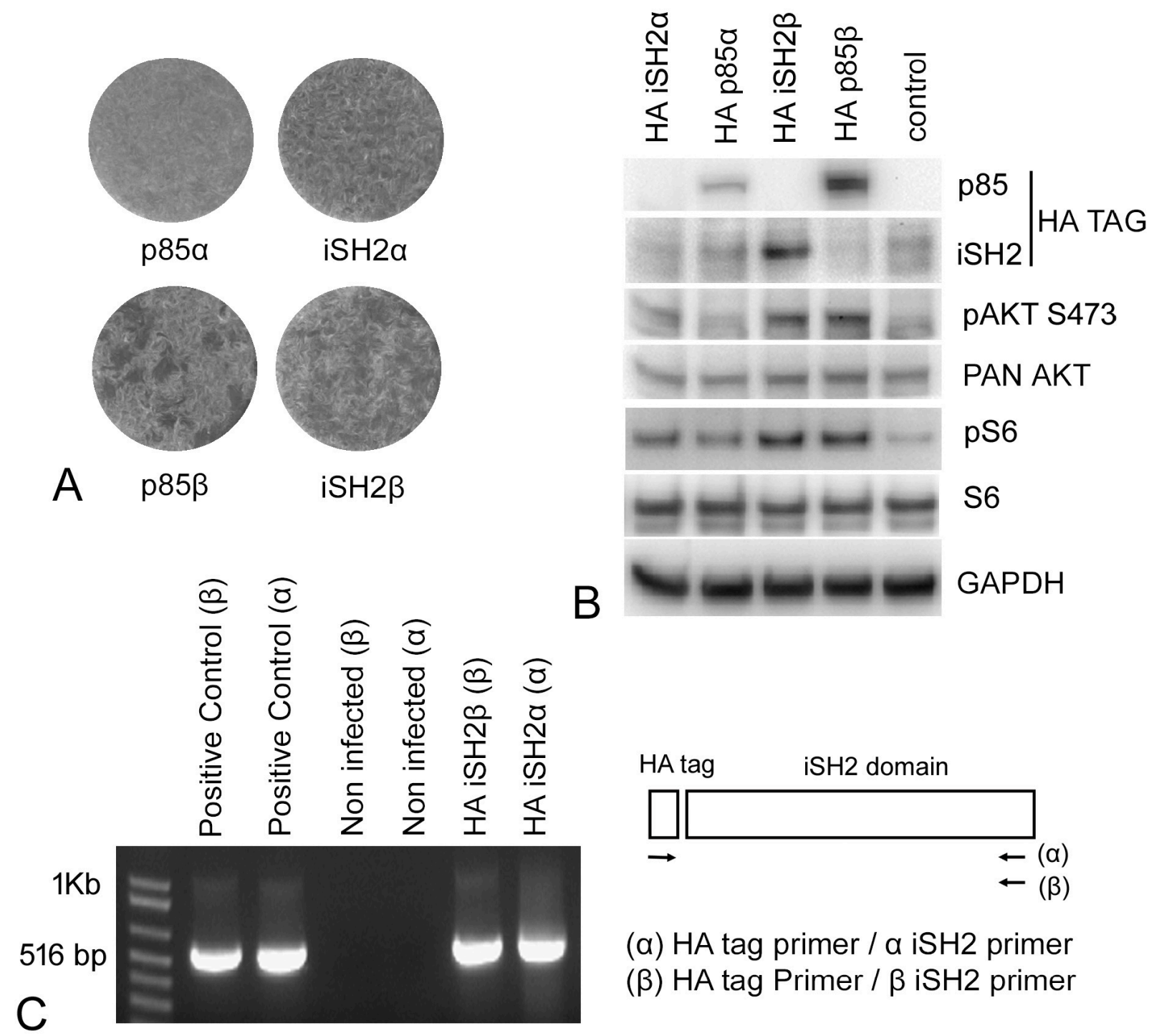

( $\alpha$ ) HA tag primer / $\alpha$ iSH2 primer
( $\beta$ ) HA tag Primer / $\beta$ iSH2 primer

Figure 7: Expression of the isolated iSH2 domains enhances oncogenic and signaling activity of PI3K. A. Representative focus assays of $\mathrm{p} 85 \alpha, \mathrm{p} 85 \beta$, iSH2 $\alpha$ and iSH2 $\beta$. Both iSH 2 domains induce a thickening of the cell sheet that is indicative of oncogenic transformation. The effect is not as pronounced as that induced by $\mathrm{p} 85 \beta$, but is evident in comparison with the non-transforming $\mathrm{p} 85 \alpha$. B. Western blots documenting enhanced PI3K signaling with pAKT S473 and pS6. Expression of the iSH2 $\alpha$ domain with the HA tag was not clearly demonstrable in standard Western blots and was therefore documented with qRTPCR C. The positive controls in this figure represent standard PCR from the DNA constructs; the HA iSH $2 \alpha$ and iSH2 $\beta$ bands were generated by qRTPCR. 


\section{Transforming activity is not correlated with protein stability}

The levels of free p85 $\beta$ in the cell are controlled by the F-box protein FBXL2 and the tyrosine protein phosphatase PTPL1 [15]. FBXL2 is a component of the SCF ubiquitin ligase complex. It binds to $\mathrm{p} 85 \beta$, mediating substrate recognition of SCF, ubiquitination and proteasomal degradation. Whereas most SCF targets require phosphorylation for recognition, in the case of $\mathrm{p} 85 \beta$, phosphorylation at Y655 inhibits the interaction with FBXL2 and increases stability. Dephosphorylation by PTPL1 enhances FBXL2 binding and degradation of $\mathrm{p} 85 \beta$. In order to explore a potential relationship between protein stability and oncogenic activity in $\mathrm{p} 85 \beta$ wild type and exchange mutants, we mutated Y 655 of p $85 \beta$ and determined the protein stabilities of these mutants and their oncogenic activities (Figures 5 and 6). The Y655A mutant, lacking the protection provided by phosphorylation, is less stable than wild type $\mathrm{p} 85 \beta$, in accord with previously published data [15]. The Y655E mutation has a stabilizing effect, apparently inhibiting FBXL2 interaction more than the phosphorylatable tyrosine (Figure 5C and 5D). The unstable Y655A mutant surprisingly retains some transforming activity in cell culture as well as signaling activity as seen by phosphorylation of AKT (Figure 5A and 5B). In contrast, the stable Y655E mutant has lost virtually all transforming and signaling activities (Figure $5 \mathrm{~A}$ and $5 \mathrm{~B})$. We then extended the protein stability studies to the cSH2 exchange constructs, first verifying that these constructs still interact with p110 (Figure 6A). The wild type $\mathrm{p} 85 \alpha$ protein is more stable than $\mathrm{p} 85 \beta$, possibly reflecting the FBXL2 interaction that is specific to the latter. The $\mathrm{p} 85 \mathrm{\beta} / \mathrm{\alpha cSH} 2$ construct is the least stable, whereas the $\mathrm{p} 85 \alpha / \beta \mathrm{cSH} 2$ construct shows about the same stability as the p $85 \beta$ wild type protein (Figure 6B and $6 \mathrm{C})$. Again, there is no correlation with oncogenic activity (compare Figure 6B and 6C with Figure 3B - 3D). The most stable protein, wild type p85 $\alpha$, lacks oncogenic activity. The two proteins with intermediate stability, wild type $\mathrm{p} 85 \beta$ and $\mathrm{p} 85 \alpha / \beta \mathrm{cSH} 2$, have the highest oncogenic activity of the four, and $\mathrm{p} 85 \beta / \alpha \mathrm{cSH} 2$ is unstable and lacks oncogenic activity. We conclude that there is no demonstrable correlation between protein stability and oncogenic activity.

\section{The iSH2 domains of $p 85 \alpha$ and $p 85 \beta$ enhance PI3K oncogenic signaling}

We cloned the p85 $\alpha$ iSH2 (iSH2 $\alpha$ ) and $p 85 \beta$ iSH2 (iSH2 $\beta$ ) domains with an N-terminal HA-tag into the RCAS(A) vector and expressed these constructs in $\mathrm{CEF}$. The expression of the iSH2 domains could not be detected by standard Western blotting using the HA-tag (Figure 7B), but was demonstrable using excessively long exposures that resulted in high background staining. We therefore verified iSH2 expression by quantitative RTPCR (Figure 7C). Expression of the iSH2 domains in CEF led to oncogenic transformation. However, compared to the activity of $\mathrm{p} 85 \beta$, the morphologic changes indicative of cellular transformation were weaker with the iSH2 domains, notably with iSH2 $\alpha$ (Figure 7A). The level of PI3K signaling associated with the expression of iSH $2 \alpha$ and iSH $2 \beta$ also showed enhancement as judged by phosphorylation of AKT at S473 and ribosomal protein $\mathrm{S} 6$ at the canonical C-terminal serine cluster (Figure 7B). Since it is known that binding of the iSH2 domains stabilizes p110 [16, 17], we examined p110 for an effect of iSH2 overexpression on protein stability using the cycloheximide chase described in Figure 6. No clear increase of p110 stability was found as a result of expressing either iSH $2 \alpha$ or iSH2 $\beta$.

\section{DISCUSSION}

The classic view of $\mathrm{p} 85$ functions is that it has two roles in the PI3K complex. Binding of $\mathrm{p} 85$ to the ABD domain stabilizes p110, and binding of the $\mathrm{nSH} 2$ domain inhibits the catalytic activity of $\mathrm{p} 110$. In vitro biochemical studies have supported these two functions extensively as have studies of mutations and truncations of PIK3R1 [1, $3,5-9,12,13,16-22]$.

In this study, we analyzed truncations, deletions and domain hybrids of $\mathrm{p} 85 \alpha$ and $\mathrm{p} 85 \beta$ for oncogenicity and signaling activity, documenting novel functional differences between the two isoforms. Our data show that all of the domains of $\mathrm{p} 85 \alpha$ and $\mathrm{p} 85 \beta$ have functional consequences on PI3K activity and oncogenic potential in the context of whole cells.

We find that the SH3 domain exerts an inhibitory effect on oncogenic potential and PI 3-kinase signaling. In the case of $\mathrm{p} 85 \alpha$, this effect could be explained by the role of the SH3 domain in p85a homodimerization which in turn is required for the interaction between p $85 \alpha$ and PTEN $[10,11,23]$. The same explanation could also apply to $\mathrm{p} 85 \beta$, although binding of this isoform to PTEN has not yet been demonstrated. Deletion of the combined SH3 and RhoGAP domains resulted in loss of oncogenic activity. The deletions at the N-terminus would also affect homodimerization of $\mathrm{p} 85 \alpha$ and possibly heterodimerization of $\mathrm{p} 85 \alpha$ and $\mathrm{p} 85 \beta[11,24]$. Interestingly, a point mutation in the RhoGAP domain has previously been shown to activate the oncogenic potential of p $85 \alpha$ [25]. The inactivity of the combined SH3-RhoGAP domain deletion is unexpected and may reflect some as yet unknown role of the RhoGAP domain in PI3K activity.

At the C-terminus of $\mathrm{p} 85$, the truncation of the cSH2 domain of $\mathrm{p} 85 \alpha$ and $\mathrm{p} 85 \beta$ revealed an unexpected difference between the two isoforms. The cSH2 domain of $\mathrm{p} 85 \alpha$ appears to have an inhibitory effect on oncogenic 
potency and signaling, whereas the $\mathrm{cSH} 2$ domain of p $85 \beta$ functions in the opposite direction: it is required for optimal transforming activity and functions as a stimulator of signaling. These opposing activities of the cSH2 domains are confirmed by domain exchange experiments: $\mathrm{p} 85 \alpha$ with the cSH2 domain of $\mathrm{p} 85 \beta$ becomes oncogenic; in contrast, $\mathrm{p} 85 \beta$ with the $\mathrm{cSH} 2$ domain of $\mathrm{p} 85 \alpha$ loses oncogenicity. These results are in contrast to the findings of Zhang et al. [13] who analyzed the structure of the p110 $\beta$-p $85 \beta$ complex and identified inhibitory contacts of the $\mathrm{p} 85 \beta \mathrm{cSH} 2$ domain and $\mathrm{p} 110 \beta$. Zhang et al. confirmed this inhibition functionally by mutating the critical contacts and observing activation of PI3K. At this time, we have no explanation for this discrepancy. The possibility that truncation of the different $\mathrm{cSH} 2$ domains affects the conformation of the residual molecules differently is unlikely in view of the data from the cSH2 domain exchanges. These suggest that the positive and negative effects of the $\mathrm{cSH} 2$ domains reflect intrinsic properties of the domains. Speculative explanations for the discordance of results include the suggestion that deletion of the $\mathrm{cSH} 2$ domain in $\mathrm{p} 85 \beta$ could induce homodimerization which would take the protein out of the PI3K signaling pathway and result in decreased oncogenic and signaling activity. The activity of the $\mathrm{p} 85 \alpha / \beta \mathrm{cSH} 2$ construct could be explained by the known preference of $\mathrm{p} 85 \alpha$ for $\mathrm{p} 110 \alpha$ where the inhibitory contacts would not exist [22]. The cSH2 domain has also been highlighted by recent genetic studies. Mutations in this domain were identified in patients with insulin resistance uncoupled from dyslipidemia [26, 27]. The PI3K-activating function of the $\mathrm{p} 85 \beta \mathrm{cSH} 2$ domain could play a significant role in determining the cancer phenotype, notably in those cancers where $\mathrm{p} 85 \beta$ is overexpressed or amplified. A special significance of the $\mathrm{p} 85 \beta \mathrm{cSH} 2$ domain is also suggested by the fact that $\mathrm{p} 85 \beta$ is only infrequently truncated in cancer, whereas such truncations are common in $\mathrm{p} 85 \alpha$ [28-30].

Larger truncations of $\mathrm{C}$-terminal domains greatly attenuate the effects of the single $\mathrm{cSH} 2$ domain deletions, resulting in constructs of low activity for $\mathrm{p} 85 \alpha$ as well as $\mathrm{p} 85 \beta$. Interacting with $\mathrm{p} 110$, the $\mathrm{nSH} 2$ domain binds to the helical domain and the iSH2 domain associates with the adapter-binding and $\mathrm{C} 2$ domains of p110 to stabilize and inhibit the catalytic subunit $[13,16,18,20,21]$. Both SH2 domains play distinct roles in the regulation of PI3K activity [17]. By binding to pYXXM motifs in upstream activating proteins, principally receptor tyrosine kinases, $\mathrm{nSH} 2$ domains facilitate a change in $\mathrm{p} 110$ to the active conformation $[6,17,19]$. The cSH2 domain of p85 $\alpha$ can also interact with IKK and PKC, resulting in phosphorylation of two serines in that domain with an inhibitory effect on PI3K signaling [31, 32]. Deletions that include the iSH2 domain would also abolish the interaction of p85 with BRD7 [33], interfering with a negative regulatory pathway of PI3K. The attenuating effect of the hinge regions in the $\mathrm{CSH} 2$ exchanges could be explained by suggesting that heterologous hinge regions might not position the cSH2 domains correctly on the recipient molecule. The activating activities observed with iSH $2 \alpha$ and iSH $2 \beta$ could result from a stabilization of p110 or from reducing the interaction with wild type p85 and hence diminishing p85-mediated inhibition. We prefer the latter explanation, because we have not detected significant stabilization of p110 in iSH2-expressing CEF.

The observation that oncogenic and signaling activities are not affected by protein stability is counterintuitive. It could reflect a low minimal concentration threshold for an oncogenic protein to score in the cell system used. That requirement could be satisfied by the high rate of protein synthesis directed by the RCAS vector. Thus, the differences in protein stability would not affect transformation or signaling.

In summary, in this study we document important and distinct properties of the $p 85 \alpha$ and $p 85 \beta$ isoforms. We confirm and extend the inhibitory function of the SH3 domain for PI3K-mediated transformation and signaling. We discover contrasting properties of the cSH2 domains and find no correlation between protein stability and oncogenicity as well as signaling activity in the investigated cell system.

\section{MATERIALS AND METHODS}

\section{Cell culture and transfection}

Chicken embryo fibroblasts (CEF) were prepared from single, pathogen-free White Leghorn embryos (Charles River, N. Franklin, CT) as previously described [34]. Transfection was performed with avian replicationcompetent retroviral vector RCAS(A) $[35,36]$ using the dimethyl sulfoxide/Polybrene method [34, 37, 38]. After two passages in the presence of serum, the cells expressing the recombinant RCAS viruses were harvested for further analysis.

\section{Oncogenic transformation}

Transformation assays were carried out in six-well plates. CEF were transfected with DNA of the recombinant RCAS(A) constructs specified below using Lipofectamine 2000 (Thermo Fisher Scientific, Asheville, NC) and were kept in growth medium for $24 \mathrm{~h}$ before being overlaid with nutrient agar containing Ham's F-10 medium with $20 \%$ Earle's balanced salt solution, $0.6 \%$ SeaPlaque Agarose, 3\% FBS, 1\% heat-inactivated chicken serum, $9 \%$ tryptose phosphate broth, $1.8 \mathrm{mM}$ glutamine, $89 \mathrm{U} /$ $\mathrm{mL}$ penicillin, $89 \mu \mathrm{g} / \mathrm{mL}$ streptomycin, $1.1 \%$ DMSO. This mixture was applied every 2-3 days for 10 days, at which point the overlay was removed and the cell layer stained 
with crystal violet for quantitative evaluation of oncogenic activity by focus formation $[39,40]$. In the figures, focus forming activities are standardized to $0.5 \mu \mathrm{g}$ DNA and are expressed relative to the focus-forming activity of $\mathrm{p} 85 \beta$. A summary of the raw data is presented in Supplementary Table S1.

\section{Constructs (See Supplementary Table S2)}

cDNA of the human PIK3R1 and PIK3R2 genes was used to construct the expression vectors using the RCAS(A) backbone [36]. The C-terminal truncations of $\mathrm{p} 85 \alpha$ and $\mathrm{p} 85 \beta$ and the $\mathrm{p} 85 \beta \mathrm{Y} 655$ mutant constructs were generated by using the QuikChange site-directed mutagenesis kit (Agilent, Wilmington, DE). For the C-terminal truncations of $\mathrm{p} 85 \beta$, amino acids G320, K435 and E600 were replaced with a stop codon (TAA); for the C-terminal truncations of $\mathrm{p} 85 \alpha$, amino acids K310, K438 and Y607 were replaced with a stop codon. For the Y655 mutants of $\mathrm{p} 85 \beta$, Y 655 was replaced with Y655A, Y655E or Y655F. Single amino acid insertions and deletions in the p85 hinge regions were generated by inserting Asp (D) at position 615 of $\mathrm{p} 85 \alpha$ and deleting D612 in $\mathrm{p} 85 \beta$. $\mathrm{N}$-terminal truncation and domain exchange mutants were constructed by PCR or fusion PCR as described [41]. For the $\mathrm{p} 85 \beta \mathrm{N}$-terminal truncations, the forward primer was modified to add a translational start site (ATG) at residue positions 105 and 314 . For the $\mathrm{p} 85 \alpha \mathrm{N}$-terminal truncations, the forward primer was modified to add a translational start site (ATG) at positions 79 and 304. Domain exchange mutants used combinations of primers to generate DNA fragments using Platinum Pfx DNA Polymerase (Invitrogen, Carlsbad, CA) (Supplementary Table 2 and Supplementary Figure 1). The primer combinations were as follows: p $85 \beta / \alpha \mathrm{cSH} 2$ (primers

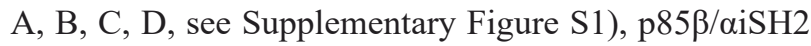
(primers A, B, E, F, G, H), p85 $\beta /$ h $\alpha \mathrm{cSH} 2$ (primers A, $\mathrm{B}, \mathrm{F}, \mathrm{H}$ ), p $85 \alpha / \beta \mathrm{cSH} 2$ (primers I, J, K, L), p $85 \alpha / \beta \mathrm{iSH} 2$ (primers I, J, M, N, O, P), p $85 \alpha / \mathrm{h} \beta \mathrm{cSH} 2$ (primers I, J, N, $\mathrm{P})$. For the HA-tagged iSH $2 \beta$ and iSH $2 \alpha$ DNA fragments were generated using Platinum Pfx DNA Polymerase (Invitrogen, Carlsbad, CA) (Supplementary Table S2). The mutated genes were subsequently cloned as SfiI DNA fragments into RCAS(A) [36]. The specified mutated p85 or wild type proteins were HA-tagged by PCR and cloned into the RCAS(A) vector. All clones were confirmed by sequencing and protein expression. N-terminal HA-tags do not affect protein function as confirmed by comparison with non-tagged protein in transformation assays.

\section{Statistical analysis}

The experiments reported here were carried out with primary cells. For repeat experiments, cells routinely have to be derived from different avian embryos. Because starting conditions are then not identical, standard statistical analyses based on mean +/- standard deviation are not applicable. However, all these tests were repeated three times with consistent results, and the figures shown are from representative experiments.

\section{RT PCR}

CEF were infected with the RCAS(A) vector expressing HA-iSH $2 \alpha$ and HA-iSH $2 \beta$ at a MOI of 1 ; non-infected CEF cells were used as negative controls. After two passages in growth medium, total cellular RNA was isolated using TRIZOL (Invitrogen, Carlsbad, CA) according to the manufacturer's protocol. First strand cDNA was synthesized using a reverse transcription kit (SuperScript III Reverse Transcriptase, Invitrogen, Carlsbad, CA) with a random primer. PCR amplification was performed using Platinum Pfx DNA Polymerase (Invitrogen, Carlsbad, CA) with a HA-tag-specific primer and iSH $2 \alpha$ and iSH2 $\beta$ C-terminal-specific sequence primers (Suplementary Table. 2). The PCR products were identified by DNA electrophoresis. The positive controls were the RCAS(A) HA-iSH $2 \alpha$ and HA-iSH2 $\beta$ vector DNA used as template in standard PCR to confirm product size.

\section{Western blots and antibodies}

CEF were infected with RCAS(A)-expressing $\mathrm{p} 85 \beta$, p $85 \alpha$, or the specified p85 mutants at a MOI of 1 . After two passages in growth medium, cells were switched for overnight incubation to Ham's F-10 medium containing $0.5 \%$ FCS (calf serum) and $0.1 \%$ chicken serum, and this was followed by $2 \mathrm{~h}$ in basal F-10. At this point, protein samples were harvested. Western blotting was performed as described [2, 42], with minor modifications. Proteins were extracted from cells using ice-cold RIPA buffer (50 $\mathrm{mM}$ Tris $\cdot \mathrm{HCl} \mathrm{pH} 8,100 \mathrm{mM} \mathrm{NaCl}, 0.5 \%$ Nonidet P-40, $0.5 \%$, sodium deoxycholate, $0.1 \%$ SDS, $1 \mathrm{mM}$ PMSF, 1 $\mathrm{mM}$ NaVO4, 1× Complete protease inhibitor mixture; Roche, Indianapolis, IN). Proteins were separated by 4-12\% gradient SDS/PAGE (Invitrogen, Carlsbad, CA) using the MOPS buffer system. Separated proteins were transferred to PVDF membranes (Millipore, Billerica, MA) with transfer buffer and a transfer apparatus (Invitrogen, Carlsbad, CA). Transferred proteins were visualized with Ponceau S staining, blocked with 5\% BSA in TBST. Primary antibodies were added as follows: p85 $\beta$ (MA1-21473, Thermo Fisher Scientific, Asheville, NC), p85a (\#4257), HA-tag (\#2367), AKT (\#4685), AKT p-S473 (\#4051), GAPDH (\#2118) (Cell Signaling, Danvers, MA). Secondary antibodies were rabbit (No. 31462) or mouse (No. 31432) anti-HRP (Thermo Scientific, Asheville, NC). Western blots were visualized using HRP conjugates, and detection was performed using 
Super Signal West Pico Chemiluminescent Substrate (Thermo Fisher Scientific, Asheville, NC) according to the manufacturer's specifications.

\section{Protein stability}

CEF were infected with RCAS(A)-expressing $p 85 \beta, p 85 \alpha, p 85 \beta$ Y $655 A, Y 655 E, Y 655 F, p 85 \beta / \alpha c S H 2$ or $\mathrm{p} 85 \alpha / \beta \mathrm{cSH} 2$ at a MOI of 1 . After two passages, the cells were treated with the translation inhibitor cycloheximide at $50 \mu \mathrm{g} / \mathrm{ml}$ to stop protein syntheses. Total protein samples were harvested using ice-cold RIPA buffer at $0,0.25,0.5$, 1,4 and $24 \mathrm{~h}$ after cycloheximide treatment for analyzing expression levels relative to GAPDH by Western blotting. We used the untreated sample as a standard. Relative protein stabilities were determined from Western blots and converted into line graphs. The error bars indicate standard deviations obtained in three independent experiments.

\section{Co-immunoprecipitation}

CEF were infected with RCAS(A) virus that expresses HA-tagged $\mathrm{p} 85 \beta$, p85 $\alpha, \mathrm{p} 85 \beta / \alpha \mathrm{cSH} 2$ or $\mathrm{p} 85 \alpha /$ $\beta \mathrm{cSH} 2$ at a MOI of 1 . After two passages, cells were rinsed with phosphate-buffered saline (PBS) and lysed in immunoprecipitation lysis buffer $(20 \mathrm{mM}$ Tris- $\mathrm{Cl}, \mathrm{pH}$ $7.4,150 \mathrm{mM} \mathrm{NaCl}, 0.5 \mathrm{mM}$ EDTA, $0.5 \%$ NP-40, $1 \mathrm{mM}$ NaVO4, $0.5 \mathrm{mM}$ phenylmethlysulfonyl fluoride (PMSF) and $1 \times$ Complete protease inhibitor mixture; Roche, Indianapolis, IN). Cell lysates ( 0.5 to $1 \mathrm{mg}$ of protein) were mixed with primary HA-tag antibody (\#2367, Cell Signaling, Danvers, MA) and incubated overnight at $4^{\circ} \mathrm{C}$ with gentle agitation. The lysates were then incubated with protein A/G beads (sc-2003, Santa Cruz Biotechnology, Santa Cruz, CA) for $1 \mathrm{~h}$ at $4^{\circ} \mathrm{C}$ with agitation. The beads were washed three times with washing buffer $(20 \mathrm{mM}$ Tris $\mathrm{pH} 7.5,100 \mathrm{mM} \mathrm{NaCl}, 0.5 \% \mathrm{NP}-40,0.5 \mathrm{mM}$ EDTA, $0.5 \mathrm{mM}$ PMSF), bound proteins were eluted by boiling in 4XSDS sample (Invitrogen, Carlsbad, CA) and analyzed for $\mathrm{p} 85-\mathrm{p} 110 \alpha$ interaction by Western blotting. The antibody against $\mathrm{p} 110$ used in the co-immunoprecipitation experiment was \#4255 from Cell Signaling, Danvers, MA.

\section{Author Contributions}

PKV designed the research. YI performed the experiments. YI, JRH and PKV analyzed the data and wrote the paper.

\section{ACKNOWLEDGMENTS}

The authors wish to express their gratitude to Lynn Ueno for outstanding technical support and to Anja Zembrzycki for superb administrative assistance.

\section{CONFLICTS OF INTEREST}

The authors have declared that no conflict of interest exists.

\section{FUNDING}

The work was supported by the National Cancer Institute under awards R35 CA197582, R01 CA153124 and R01 CA151574. This is manuscript 29410 of The Scripps Research Institute.

\section{REFERENCES}

1. Burke JE, Williams RL. Synergy in activating class I PI3Ks. Trends Biochem Sci. 2015; 40:88-100.

2. Ito Y, Hart JR, Ueno L, Vogt PK. Oncogenic activity of the regulatory subunit p85beta of phosphatidylinositol 3-kinase (PI3K). Proc Natl Acad Sci U S A. 2014; 111:16826-16829.

3. Mellor P, Furber LA, Nyarko JN, Anderson DH. Multiple roles for the $\mathrm{p} 85 \mathrm{alph}$ isoform in the regulation and function of PI3K signalling and receptor trafficking. Biochem J. 2012; 441:23-37.

4. Thorpe LM, Spangle JM, Ohlson CE, Cheng H, Roberts TM, Cantley LC, Zhao JJ. PI3K-p110alpha mediates the oncogenic activity induced by loss of the novel tumor suppressor PI3K-p85alpha. Proc Natl Acad Sci U S A. 2017; 114:7095-7100.

5. Walker EH, Perisic O, Ried C, Stephens L, Williams RL. Structural insights into phosphoinositide 3-kinase catalysis and signalling. Nature. 1999; 402:313-320.

6. Wu H, Yan Y, Backer JM. Regulation of class IA PI3Ks. Biochem Soc Trans. 2007; 35:242-244.

7. Backer JM. The regulation of class IA PI 3-kinases by intersubunit interactions. Curr Top Microbiol Immunol. 2010; 346:87-114.

8. Vadas O, Burke JE, Zhang X, Berndt A, Williams RL. Structural basis for activation and inhibition of class I phosphoinositide 3-kinases. Sci Signal. 2011; 4:re2.

9. Burke JE, Williams RL. Dynamic steps in receptor tyrosine kinase mediated activation of class IA phosphoinositide 3-kinases (PI3K) captured by H/D exchange (HDX-MS). Adv Biol Regul. 2013; 53:97-110.

10. Chagpar RB, Links PH, Pastor MC, Furber LA, Hawrysh AD, Chamberlain MD, Anderson DH. Direct positive regulation of PTEN by the $\mathrm{p} 85$ subunit of phosphatidylinositol 3-kinase. Proc Natl Acad Sci U S A. 2010; 107:5471-5476.

11. Cheung LW, Walkiewicz KW, Besong TM, Guo H, Hawke DH, Arold ST, Mills GB. Regulation of the PI3K pathway through a p85alpha monomer-homodimer equilibrium. Elife. 2015; 4:e06866.

12. Hofmann BT, Jucker M. Activation of PI3K/Akt signaling by n-terminal $\mathrm{SH} 2$ domain mutants of the p85alpha 
regulatory subunit of PI3K is enhanced by deletion of its c-terminal SH2 domain. Cell Signal. 2012; 24:1950-1954.

13. Zhang X, Vadas O, Perisic O, Anderson KE, Clark J, Hawkins PT, Stephens LR, Williams RL. Structure of lipid kinase p110beta/p85beta elucidates an unusual SH2domain-mediated inhibitory mechanism. Mol Cell. 2011; 41:567-578.

14. Dhand R, Hiles I, Panayotou G, Roche S, Fry MJ, Gout I, Totty NF, Truong O, Vicendo P, Yonezawa K, Kasuga M, Courtneidge SA, Waterfield MD. Rapamycin-induced miR21 promotes mitochondrial homeostasis and adaptation mTORC1 activated cells. PI 3-kinase is a dual specificity enzyme: autoregulation by an intrinsic protein-serine kinase activity. EMBO J. 1994; 13:522-533.

15. Kuchay S, Duan S, Schenkein E, Peschiaroli A, Saraf A, Florens L, Washburn MP, Pagano M. FBXL2- and PTPL1mediated degradation of p110-free p85beta regulatory subunit controls the PI(3)K signalling cascade. Nat Cell Biol. 2013; 15:472-480.

16. Wu H, Shekar SC, Flinn RJ, El-Sibai M, Jaiswal BS, Sen KI, Janakiraman V, Seshagiri S, Gerfen GJ, Girvin ME, Backer JM. Regulation of Class IA PI 3-kinases: C2 domain-iSH2 domain contacts inhibit p85/p110alpha and are disrupted in oncogenic p85 mutants. Proc Natl Acad Sci U S A. 2009; 106:20258-20263.

17. Yu J, Wjasow C, Backer JM. Regulation of the p85/ p110alpha phosphatidylinositol 3'-kinase. Distinct roles for the n-terminal and c-terminal SH2 domains. J Biol Chem. 1998; 273:30199-30203

18. Amzel LM, Huang CH, Mandelker D, Lengauer C, Gabelli SB, Vogelstein B. Structural comparisons of class I phosphoinositide 3-kinases. Nat Rev Cancer. 2008; 8:665669.

19. Cantley LC. The phosphoinositide 3-kinase pathway. Science. 2002; 296:1655-1657.

20. Echeverria I, Liu Y, Gabelli SB, Amzel LM. Oncogenic mutations weaken the interactions that stabilize the p110alpha-p85alpha heterodimer in phosphatidylinositol 3-kinase alpha. FEBS J. 2015; 282:3528-3542.

21. Huang CH, Mandelker D, Gabelli SB, Amzel LM. Insights into the oncogenic effects of PIK3CA mutations from the structure of p110alpha/p85alpha. Cell Cycle. 2008; 7:11511156.

22. Sun M, Hillmann P, Hofmann BT, Hart JR, Vogt PK. Cancer-derived mutations in the regulatory subunit p85alpha of phosphoinositide 3-kinase function through the catalytic subunit p110alpha. Proc Natl Acad Sci U S A. 2010; 107:15547-15552.

23. Anderson DH. p85 plays a critical role in controlling flux through the PI3K/PTEN signaling axis through dual regulation of both p110 (PI3K) and PTEN. Cell Cycle. 2010; 9:2055-2056.

24. Park SW, Zhou Y, Lee J, Lu A, Sun C, Chung J, Ueki K, Ozcan U. The regulatory subunits of PI3K, p85alpha and p85beta, interact with XBP-1 and increase its nuclear translocation. Nat Med. 2010; 16:429-437.

25. Chamberlain MD, Chan T, Oberg JC, Hawrysh AD, James KM, Saxena A, Xiang J, Anderson DH. Disrupted RabGAP function of the p85 subunit of phosphatidylinositol 3-kinase results in cell transformation. J Biol Chem. 2008; 283:15861-15868.

26. Huang-Doran I, Tomlinson P, Payne F, Gast A, Sleigh A, Bottomley W, Harris J, Daly A, Rocha N, Rudge S, Clark J, Kwok A, Romeo S, et al. Insulin resistance uncoupled from dyslipidemia due to C-terminal PIK3R1 mutations. JCI Insight. 2016; 1:e88766.

27. Winnay JN, Solheim MH, Dirice E, Sakaguchi M, Noh HL, Kang HJ, Takahashi H, Chudasama KK, Kim JK, Molven A, Kahn CR, Njolstad PR. PI3-kinase mutation linked to insulin and growth factor resistance in vivo. J Clin Invest. 2016; 126:1401-1412.

28. Gao J, Aksoy BA, Dogrusoz U, Dresdner G, Gross B, Sumer SO, Sun Y, Jacobsen A, Sinha R, Larsson E, Cerami E, Sander C, Schultz N. Integrative analysis of complex cancer genomics and clinical profiles using the cBioPortal. Sci Signal. 2013; 6:pl1.

29. Cerami E, Gao J, Dogrusoz U, Gross BE, Sumer SO, Aksoy BA, Jacobsen A, Byrne CJ, Heuer ML, Larsson E, Antipin Y, Reva B, Goldberg AP, et al. The cBio cancer genomics portal: an open platform for exploring multidimensional cancer genomics data. Cancer Discov. 2012; 2:401-404.

30. Zehir A, Benayed R, Shah RH, Syed A, Middha S, Kim HR, Srinivasan P, Gao J, Chakravarty D, Devlin SM, Hellmann MD, Barron DA, Schram AM, et al. Mutational landscape of metastatic cancer revealed from prospective clinical sequencing of 10,000 patients. Nat Med. 2017; 23:703-713.

31. Lee JY, Chiu YH, Asara J, Cantley LC. Inhibition of PI3K binding to activators by serine phosphorylation of PI3K regulatory subunit p85alpha Src homology-2 domains. Proc Natl Acad Sci U S A. 2011; 108:14157-14162.

32. Comb WC, Hutti JE, Cogswell P, Cantley LC, Baldwin AS. p85alpha SH2 domain phosphorylation by IKK promotes feedback inhibition of PI3K and Akt in response to cellular starvation. Mol Cell. 2012; 45:719-730.

33. Chiu YH, Lee JY, Cantley LC. BRD7, a tumor suppressor, interacts with p85alpha and regulates PI3K activity. Mol Cell. 2014; 54:193-202.

34. Duff RG, Vogt PK. Characteristics of two new avian tumor virus subgroups. Virology. 1969; 39:18-30.

35. Hughes SH, Greenhouse JJ, Petropoulos CJ, Sutrave P. Adaptor plasmids simplify the insertion of foreign DNA into helper-independent retroviral vectors. J Virol. 1987; 61:3004-3012.

36. Aoki M, Batista O, Bellacosa A, Tsichlis P, Vogt PK. The akt kinase: molecular determinants of oncogenicity. Proc Natl Acad Sci U S A. 1998; 95:14950-14955.

37. Kawai S, Nishizawa M. New procedure for DNA transfection with polycation and dimethyl sulfoxide. Mol 
Cell Biol. 1984; 4:1172-1174.

38. Chang HW, Aoki M, Fruman D, Auger KR, Bellacosa A, Tsichlis PN, Cantley LC, Roberts TM, Vogt PK. Transformation of chicken cells by the gene encoding the catalytic subunit of PI 3-kinase. Science. 1997; 276:18481850.

39. Zhao L, Vogt PK. Helical domain and kinase domain mutations in p110alpha of phosphatidylinositol 3-kinase induce gain of function by different mechanisms. Proc Natl Acad Sci U S A. 2008; 105:2652-2657.

40. Sun M, Hart JR, Hillmann P, Gymnopoulos M, Vogt PK. Addition of $\mathrm{N}$-terminal peptide sequences activates the oncogenic and signaling potentials of the catalytic subunit p110alpha of phosphoinositide-3-kinase. Cell Cycle. 2011; 10:3731-3739.
41. Atanassov II, Atanassov II, Etchells JP, Turner SR. A simple, flexible and efficient PCR-fusion/Gateway cloning procedure for gene fusion, site-directed mutagenesis, short sequence insertion and domain deletions and swaps. Plant Methods. 2009; 5:14.

42. Aoki M, Schetter C, Himly M, Batista O, Chang HW, Vogt PK. The catalytic subunit of phosphoinositide 3-kinase: requirements for oncogenicity. J Biol Chem. 2000; 275:6267-6275. 